\title{
Rapid detection of Pfcrt and Pfmdr I mutations in Plasmodium falciparum isolates by FRET and in vivo response to chloroquine among children from Osogbo, Nigeria
}

\author{
Olusola Ojurongbe ${ }^{1,2,4}$, Titus O Ogungbamigbe 3 , Adetayo F Fagbenro- \\ Beyioku ${ }^{4}$, Rolf Fendel ${ }^{1}$, Peter G Kremsner ${ }^{1}$ and Jürgen FJ Kun*1
}

Address: ${ }^{1}$ Department of Parasitology, Institute of Tropical Medicine, University of Tübingen, Wilhelmstr. 27, 72074 Tübingen, Germany, ${ }^{2}$ Department of Medical Microbiology \& Parasitology College of Health Sciences, Ladoke Akintola University of Technology, Osogbo, Nigeria, ${ }^{3}$ Department of Pharmacology \& Therapeutics College of Health Sciences, Ladoke Akintola University of Technology, Osogbo, Nigeria and ${ }^{4}$ Department of Medical Microbiology and Parasitology College of Medicine, University of Lagos, Nigeria

Email: Olusola Ojurongbe - stojurongbe@yahoo.com; Titus O Ogungbamigbe - bisiogun1562@yahoo.com; Adetayo F FagbenroBeyioku - afbeyioku@yahoo.com; Rolf Fendel -rolf_fendel@yahoo.de; Peter G Kremsner - peter.kremsner@uni-tuebingen.de; Jürgen FJ Kun* - juergen.kun@uni-tuebingen.de

* Corresponding author

Published: II April 2007

Malaria Journal 2007, 6:41 doi:|0.1|186/|475-2875-6-4I
Received: 20 December 2006

Accepted: II April 2007

This article is available from: http://www.malariajournal.com/content/6/l/4 I

(c) 2007 Ojurongbe et al; licensee BioMed Central Ltd.

This is an Open Access article distributed under the terms of the Creative Commons Attribution License (http://creativecommons.org/licenses/by/2.0), which permits unrestricted use, distribution, and reproduction in any medium, provided the original work is properly cited.

\begin{abstract}
Background: Chloroquine (CQ) has been in use in Africa for a long time. Because of misuse, this drug has now lost its efficacy due to the emergence of resistance strains in most parts of Africa. Recently, it was shown that after chloroquine has been withdrawn from the market, chloroquine-sensitive Plasmodium falciparum re-emerged and chloroquine could again be used successfully as an antimalarial. Surveillance of parasite populations is, therefore, important to decide whether chloroquine could be re-introduced.

Methods: To estimate the prevalence of the most pivotal polymorphisms, including Pfcrt K76T, Pfmdrl N86Y and Pfmdrl YI84F mutations, and their contributions to the outcome of CQ treatment, isolates from Osogbo Western Nigeria were tested using the Fluorescence Resonance Energy Transfer (FRET) method on a real-time PCR instrument.

Results: 116 children with acute uncomplicated $P$. falciparum malaria infections were treated with the standard dosage of $\mathrm{CQ}$ and followed-up for 28 days. Blood samples were collected on filter paper at enrollment and during follow-up for identification of parasite carrying the chloroquine resistant transporter ( $p f c r t)$ and P. falciparum-multi drug resistance ( $p f m d r l)$ gene mutations. Parasitological assessment of response to treatment showed that $62 \%$ of the patients were cured and $38 \%$ failed the CQ treatment. The presence of single mutant pfcrt $(T 76)$ alleles $(P=0.003)$ and in combination with mutant pfmdrl Y86 ( $P=0.028)$ was significantly associated with in vivo CQR. No other mutation on its own or in combinations was significantly associated with treatment outcome. Mutant pfcrt was more prevalent in both pre- and post-treatment isolates. No association was observed between age or initial level of parasitaemia and chloroquine treatment outcome.
\end{abstract}

Conclusion: The result established the usefulness and accuracy of real time PCR in pfcrt and pfmdrl mutation detection and also give further evidence to the reliability of the pfort T76 point mutation as a molecular marker for CQ resistance. 


\section{Background}

While there is an active search for new antimalarial drug combinations that could prevent or delay further spread of resistance, there is a need to understand the basis of parasites resistance to chloroquine (CQ) and other antimalarial drugs and explore potentials to use the data in improving the potency and rational for selecting components for effective drug combination. Constant observation of the existing parasite population concerning their genetic makeup determining the resistance to CQ became even more important since it was shown that after CQ withdrawal for therapy CQ-sensitive parasite re-occurred [1]. So written-off drugs may come into focus again.

The molecular basis of CQ resistance in Plasmodium falciparum is still unclear, and the association of point mutations in different genes with chloroquine-resistance has been largely studied in the last decade. In 2000, pfcrt gene was identified [2]. This gene consisting of 13 exons showed 6-8 point mutations including one that appears to play a crucial role in CQR [3]. A lysine to threonine change at position 76 (K76T) which was subsequently found in every in vitro CQR parasite from around the world $[4,5]$ was identified as an important mutation associated with CQR. The resistance was associated with a reduced accumulation of CQ in the parasite digestive vacuole but how the pfcrt gene exerts such an effect on the digestive vacuole is still unclear. Many studies have shown that the $p f c r t$ play a crucial on CQR, but this mutation was not the sole requirement, suggesting that other factors including host factors are responsible for the clearance of CQR parasites [6].

Polymorphisms in pfmdr1, a gene located on chromosome 5 which encodes the P. falciparum P-glycoprotein homologue-1 is also thought to modulate CQR. It is a typical member of the ATP-binding cassette transporter superfamily localized in the parasite vacuole, where it may regulate intracellular drug concentrations [7]. Mutations were observed at the amino acids 86, 184, 1034, 1042 , and 1246, which were strongly linked to the CQR in laboratory clones obtained from various regions [8]. However, the link between $p f m d r 1$ and CQR still remains unclear and controversial $[6,9]$. While some field studies had indicated that there is positive association between CQR and mutation (asparagine to tyrosine change) at position $86(\mathrm{~N} 86 \mathrm{Y})[10,11]$, several others had doubts about this association $[12,13]$. Currently, pfmdr1 mutations are said to assist the CQR parasites by augmenting the level of resistance. A combination of pfcrt and $p f m d r 1$ polymorphisms is believed to result in higher levels of CQR $[4,7]$.

In Nigeria, CQ has been used for many years as the firstline treatment for uncomplicated malaria. However, like many other malaria endemic regions the therapeutic efficacy of CQ has decreased considerably. This, therefore, has led to the change in the first line drug for the treatment of malaria to artemisinin-based combination, although, CQ is still widely used in the country. In order to explore the roles of $p f c r t$ and $p f m d r 1$ polymorphisms in CQR, the Fluorescence Resonance Energy Transfer (FRET) method was used to determine these polymorphisms and their in vivo sensitivity to chloroquine in $P$. falciparum isolates from Osogbo Western Nigeria. The use of a Real Time PCR assay for a rapid, sensitive, and specific detection of these mutations was also assessed.

\section{Materials and methods Study site and patients}

The study was undertaken between July 2004 and January 2005 in the town of Osogbo located in the western part of Nigeria. Osogbo is the state capital of Osun state Nigeria and it represents a typical urban setting in Nigeria. Malaria is present throughout the year with a marked increase during the raining season (i.e. April - September). Febrile children (1-12 years old) attending the Osun state Hospital and Ladoke Akintola University Teaching hospital were screened for $P$. falciparum parasitaemia. Blood films were stained with $10 \%$ Giemsa and examined microscopically. Criteria for recruitment in this study were: (1) asexual parasitaemia between $2,000 / \mu \mathrm{l}$ and $200,000 / \mu \mathrm{l}$, (2) no signs of severity or severe malaria (including severe anaemia defined by haemoglobin $<5 \mathrm{~g} / \mathrm{dl}$ ), (3) no intake of antimalarial drugs during the preceding four weeks, (4) informed consent from the patient parent or guardian. The detected parasitaemic cases were treated with $25 \mathrm{mg} /$ $\mathrm{kg}$ chloroquine in divided doses for three days at $10 \mathrm{mg} /$ $\mathrm{kg}$ daily for D0 and D1 and $5 \mathrm{mg} / \mathrm{kg}$ for D2. Subsequent follow up appointments were scheduled for days 3, 7, 14, 21 and 28.

Classification of responses to treatment was done according to the WHO criteria [14]. The cure rate on day 28 of the follow-up was defined as the percentage of children who remained free of parasites. Two drops of blood were also blotted onto $3 \mathrm{MM}$ Whatman filter paper on days 0 before treatment and during following up when there was re-occurrence of clinical symptoms for extraction and analysis of parasites DNA. Treatment failure rates were corrected by $m s p-2$ genotyping of parasites at enrollment and recrudescence of infections [15]. The study received ethical approval from the ethical review committee boards of the joint College of Health Sciences/Ladoke Akintola University Teaching Hospital and Osun State Hospitals Management Board.

Detection of Pfort and Pfmdrl mutations by Real time PCR Parasite genomic DNA was extracted from blood samples collected on filter paper using a QIAamp DNA blood kit 
(Qiagen, Hilden, Germany), according to the manufacturer's instructions. The oligonucleotide probes and primer used to detect the polymorphisms in pfcrt and pfmdr1 are shown in Table 1.

For $p f c r t$ analysis the sensor probe labeled with fluorescein at the 3 ' end is designed to be perfectly complementary to the mutation site. An amplification primer iLC labeled with Cy 5 on the third base from the 3 'end is used as a reverse primer which is extended during amplification. During FRET, fluorescein which is excited by the light source of the Rotor Gene instrument transfers its energy to the Cy5 incorporated into the PCR product working as anchor probe $[16,17]$. A specific melting temperature is then obtained for each genotype: a sensor probe spanning one mismatch could still hybridize to the target sequence but will melt off at lower temperature than a sensor probe with a perfect match. Primers and probes for $p f c r t$ were designed and synthesized by TIB MOLBIOL (DNA synthesis service, Berlin, Germany)

For $p f m d r 1$ mutations, hybridization probes consisted of two different oligonucleotides that bind to an internal sequence amplified by forward and reverse primers Table 1. The sensor probe, labeled at the 3'end with FAM, is designed to match the mutation sites. The anchor probe, labeled at the 5' end with Cy5 and phosphorylated at the 3 ' end to prevent extension by Taq polymerase, is designed to conserved sequences adjacent to the mutation sites. Both probes, localised on the same DNA strand, could hybridize in a head-to tail arrangement, bringing the two fluorescent dyes into close proximity. During FRET, FAM was excited by the light source of the Rotor Gene instrument. The excitation energy was transferred to the acceptor fluorophore, Cy5, and the emitted fluorescence was measured on the Rotor Gene channel in continuous during the melting phase. A specific melting temperature is then obtained for each genotype as described above. Pfmdr1 primers and probes were synthesized by Operon Biotechnology (Cologne, Germany).

\section{PCR amplification}

Amplification was performed with Rotor Gene 3000 (Corbbett, Sydney, Australia). For pfort forward primer was added at a final concentration of $0.4 \mu \mathrm{M}$, iLC primer $0.5 \mu \mathrm{M}$ and probe at $0.2 \mu \mathrm{M}$. The amplification program consisted of an initial step at $95^{\circ} \mathrm{C}$ for $10 \mathrm{~min}$, amplification was performed with 40 cycles of denaturation $\left(95^{\circ} \mathrm{C}\right.$ for $10 \mathrm{~s})$, annealing $\left(50^{\circ} \mathrm{C}\right.$ for $\left.15 \mathrm{~s}\right)$, and extension $\left(65^{\circ} \mathrm{C}\right.$ for $15 \mathrm{~s}$ ). The melting curve program consisted of one cycle of $95^{\circ} \mathrm{C}$ for $15 \mathrm{~s}$, and heating at $36^{\circ} \mathrm{C}$ to $75^{\circ} \mathrm{C}$ rising by $1^{\circ} \mathrm{C}$.

For $p f m d r 1$, the master mix contains a final concentration of $0.4 \mu \mathrm{M}$ of both primers and $0.2 \mu \mathrm{M}$ of both Anchor and sensor probes. For $p f m d r 1$ codons 86 and 184 , the PCR program was as follows: $5 \mathrm{~min}$ at $95^{\circ} \mathrm{C}, 40$ cycles of $94^{\circ} \mathrm{C}$ for $10 \mathrm{~s}, 52^{\circ} \mathrm{C}$ for $30 \mathrm{~s}$, and $72^{\circ} \mathrm{C}$ for $40 \mathrm{~s}$. The melting program consisted of one cycle of $95^{\circ} \mathrm{C}$ for $15 \mathrm{~s}$ and heating from 36 to $85^{\circ} \mathrm{C}$ rising by $1^{\circ} \mathrm{C}$.

\section{Statistical analysis}

For analysis purposes, each isolate was classified based on the presence or absence of a resistance-associated allele and infections with mixed wild-type/mutant alleles of $p f c r t$ or $p f m d r 1$ were treated as mutants. Data were analysed using the statistical programs JMP for Windows. For univariate analysis, frequencies were compared using the Fisher's exact tests. Two sided $P$ values $<0.05$ indicated statistical significance. McNemar's test was used to compare the samples before and after treatment.

\section{Results}

\section{Patient treatment outcome}

The potential for detection of a point mutation in $p f c r t$ and $p f m d r 1$ genes using hybridization probes on Rotor Gene technology has been evaluated. DNA from 116 samples of patients treated with chloroquine and successfully followed up for 28 days were evaluated for $p f c r t$ and Pfmdr1 (86 and 184) mutations.

Of these, 69 (59\%) were males and 47 (41\%) were females. The mean age of the children was $46 \pm 36$ months (6-144 months). Geometric mean parasite density was 9,061 parasites/ $\mu$ l of blood on the day of enrollment. While infections in $72(62 \%)$ of the patients were cured with a standard dosage of CQ, 44 (38\%) failed to respond to treatment. The clinical data and therapeutic responses of the patients stratified by age is shown in Table 2 .

\section{Real-time PCR for Pfcrt and Pfmdrl gene detection in blood samples}

Wild-type and mutant alleles were rapidly determined by comparing the melting temperature for the alleles with the melting temperature of the reference alleles obtained by the FRET assay. The reference strains used for this study were 3D7, FCR, S007 and K1 laboratory strains which were all sequenced to determine the mutations that were present. A confirmed field sample of Plasmodium malariae was used as control along with no template. P. falciparum strain K1 parasites were kindly provided by Katja Becker (Justus-Liebig-University Giessen). Both 3D7 and FCR showed the wild type sequence for $p f c r t$ (K76) and Pfmdr1 (N86 and Y184). K1 showed mutant nucleotide at position 76 (T76) for Pfcrt and position 86 (Y86) for $p f m d r 1$ while $\mathrm{S} 007$ showed mutation at positions 76 for $p f c r t, 86$ and 184 for $p f m d r 1$. DNA yielded a specific melting temperature of $46.5 \pm 0.3^{\circ} \mathrm{C}$ for the pfcrt mutant alleles and $65.3 \pm 0.4$ for the wild alleles. The melting temperatures 
Table I: Sequence of primers and probes used for Pfcrt and PfmdrI amplification and melting temperatures of the sensor probes of each allele

Sequence 5' to $\mathbf{3}^{\prime}$
Pfcrt
Forward Primer: CTTGTCTTGGTAAATGTGCTCA
iLC Primer: GTTACCAATTTTGTTTAAAGTTCT
Sensor Probe: TGTGTAATTGAAACAATTTTTGCTAA

Melting temperatures $\left({ }^{\circ} \mathrm{C}\right)$ of the sensor probes Wild Mutant

Pfmdr I

Forward Primer: TGTATTATCAGgAGgAACATTACC

Reverse Primer: ACCACCAAACATAAATTAACGGA

Sensor Probe 86: ATTAATATCATCATAAATACATG

Anchor Probe 86: TCTTTAATATTACACCAAACACAGATAT

Sensor Probe 184: TAAAAAATGCACGTTTGACTTTATGTATTA

Anchor Probe 184: CCTTTTTAGGTTTATTTATTTGGTCAT

$\begin{array}{ll}51.8 \pm 0.3 & 56.5 \pm 0.2 \\ 53.0 \pm 0.2 & 58.7 \pm 0.3\end{array}$

for all the alleles are shown in Table 1. The melting analysis is shown in Figures $1 \& 2$

\section{Association between Pfcrt and PfmdrI mutations and chloroquine treatment failure}

The prevalence of Pfcrt T76 and Pfmdr1 (Y86 and F184) mutations known to be involved in CQR resistance in West Africa was evaluated. By in-vivo testing, 38\% of the $P$. falciparum isolates were chloroquine resistant, although by molecular analysis $74 \%$ of the pre-treatment isolates carried the mutant pfcrt T76 and $14 \%$ had mixed $(\mathrm{T} 76+\mathrm{K} 76)$ allele that has been associated with chloroquine resistance in other studies $[6,18]$. The pfmdr1 Y86 and F184 alleles were present in $29 \%$ and $64 \%$ of the pretreatment isolates, respectively (Table 3 ).

The hypothesis whether allelic variations in pfcrt and pfmdr1 of $P$. falciparum isolates are associated with CQ treatment outcome was tested in the study area. The presence of $p f c r t$ or $p f m d r 1$ mutations or the combination of these mutations in samples collected before chloroquine treatment was examined for their association with patient treatment outcome. The $p f c r t \mathrm{~K} 76 \mathrm{~T}$ allele was significantly associated with CQ treatment failure $(\mathrm{P}=0.003)$. Both pfmdr1 N86Y and Y184F did not show any significant association. The combination of pfcrt 76 and pfmdr1 86 also produces weak significant association $(\mathrm{p}=0.028)$ (Table 3).

For analysis of parasites strains before and after treatment, 44 pairs was collected; each consisting of the parasite strain before treatment and that from the recrudescence infection. All the 44 samples had either mutant or mixed T76 allele in their pre-treatment sample and 43/44 (98\%) had the mutant pfcrt allele after treatment. Only one isolate showed the wild $p f c r t$ allele and genotyping by MSP2 confirmed the isolate to be a new infection. For Pfmdr1 Y86 and F184 the prevalence of the alleles at pre-treatment isolates were $17 \%$ and $33 \%$, respectively, and at recrudescence the prevalence of Y 86 was $18 \%$ while that of F184 was 27\%. The analysis of nonresponding and recrudescent parasites showed a pronounced significant difference between the samples collected before and after chemotherapy for the isolates having pfmdr1 F184 mutation ( $\mathrm{p}=0.031)$ while for other alleles tested there was no

Table 2: Enrollment clinical data and therapeutic responses of patients with acute uncomplicated Plasmodium falciparum treated with chloroquine stratified by age

\begin{tabular}{|c|c|c|c|c|c|c|c|c|c|c|c|c|}
\hline \multirow{2}{*}{$\begin{array}{l}\text { Age } \\
\text { (Yrs) }\end{array}$} & \multirow[t]{2}{*}{ Freq } & \multicolumn{2}{|c|}{ Sex } & \multirow{2}{*}{$\begin{array}{l}\text { Temp } \\
\text { (SD) }\end{array}$} & \multirow{2}{*}{$\begin{array}{c}\text { Mean } \\
\text { PD }\end{array}$} & \multirow{2}{*}{$\begin{array}{l}\text { Mean } \\
\text { FC }\end{array}$} & \multirow{2}{*}{$\begin{array}{c}\text { Mean } \\
\text { PC }\end{array}$} & \multirow{2}{*}{$\begin{array}{l}\text { T76 } \\
(\%)\end{array}$} & \multirow{2}{*}{$\begin{array}{l}\text { Y86 } \\
\text { (\%) }\end{array}$} & \multirow{2}{*}{$\begin{array}{c}\text { FI } 84 \\
(\%)\end{array}$} & \multicolumn{2}{|c|}{ CQ Treatment } \\
\hline & & $\mathbf{M}$ & $\mathbf{F}$ & & & & & & & & Cured & Failed \\
\hline $0<4$ & 72 & 39 & 33 & $37.7( \pm 1.13)$ & 8261.066 & 2.7 & 2.6 & 67 (93\%) & $27(38 \%)$ & 49 (68\%) & $46(58 \%)$ & $26(36 \%)$ \\
\hline $4<8$ & 27 & 20 & 7 & $38.0( \pm 1.3)$ & 11632.6 & 1.2 & 2.4 & $22(81 \%)$ & $9(33 \%)$ & $20(74 \%)$ & 17 (63\%) & $10(37 \%)$ \\
\hline $8-12$ & 17 & 10 & 7 & $37.8( \pm 0.84)$ & 9015.282 & 1.3 & 2.5 & $16(94 \%)$ & $6(35 \%)$ & 12 (7I\%) & $9(53 \%)$ & $8(47 \%)$ \\
\hline Total & 116 & 69 & 47 & $37.8( \pm 1.3)$ & 9061.375 & 1.7 & 2.5 & $102(88 \%)$ & $42(36 \%)$ & 81 (70\%) & $72(62 \%)$ & 44 (38\%) \\
\hline
\end{tabular}

Key: Freq $=$ Frequency

$\mathrm{SD}=$ Standard deviation

$\mathrm{PD}=$ Parasite density on Day 0

$\mathrm{FC}=$ Fever clearance time in days

$\mathrm{PC}=$ Parasite clearance time in days 


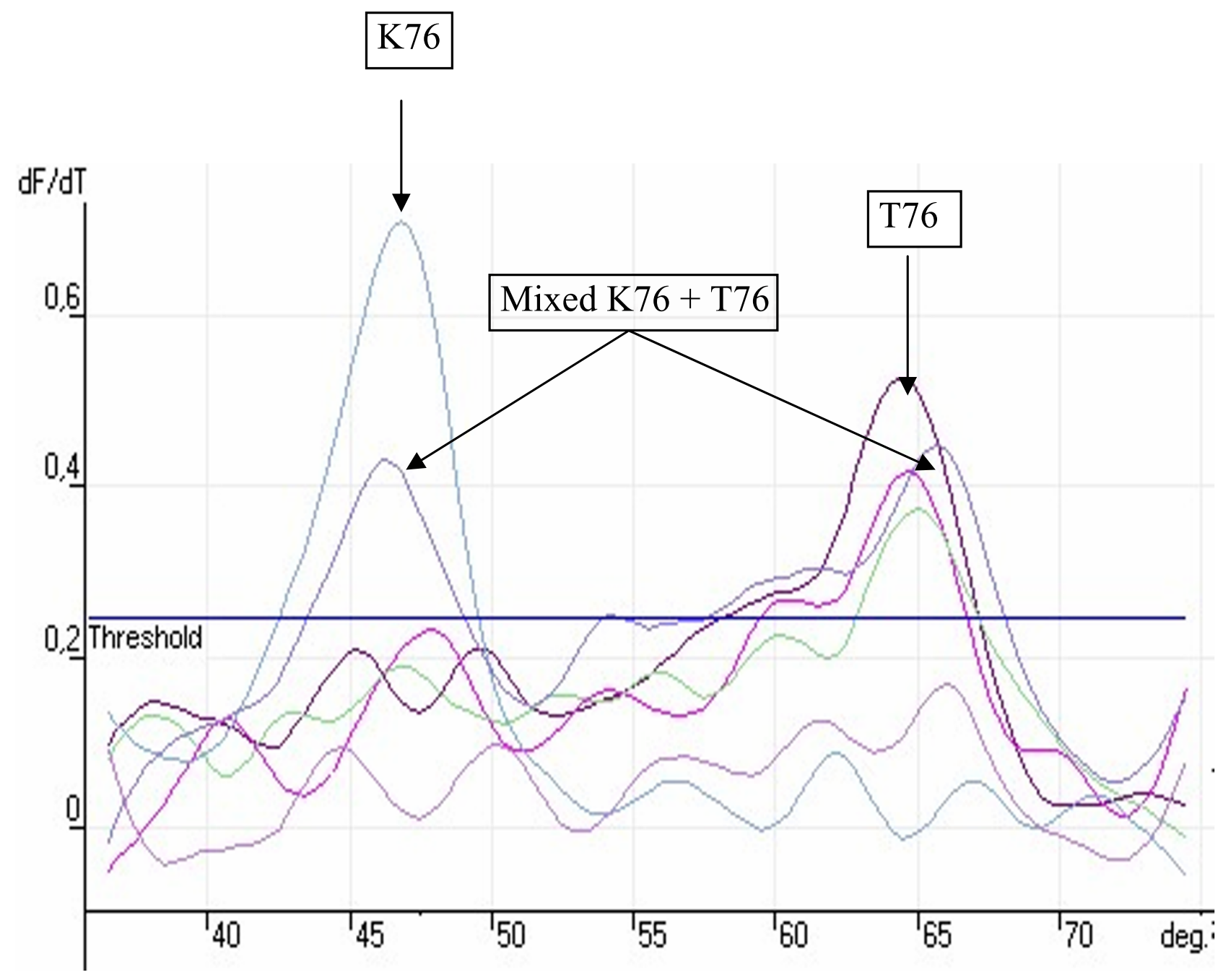

Figure I

Example of a typical real time rum showing melting curve analysis of Pfcrt position 76.

significant difference between the pre and post treatment samples Table 4.

\section{Discussion}

In this study, a Real Time PCR assay for the detection of $p f c r t$ and $p f m d r 1$ alleles thought to be associated with CQ susceptibility and resistance was described. This RealTime PCR assay was demonstrated to be rapid, sensitive, and specific for the detection and characterization of $P$. falciparum genetic marker of CQR. The assay detected mixed alleles infections and clearly discriminated between CQ-susceptible and CQ-resistant isolates. Its speed (up to 72 samples can be assayed in a 2-h experiment) and performance characteristics may eliminate the need for more complicated approaches and make it an attractive strategy that could easily be adapted to largescale studies of drug resistance.

Point mutations in the pfcrt gene and to a lesser extent, in the pfmdr1 gene are thought to be associated with CQR $[2,6]$. The goal of this study was to evaluate the utility of these molecular markers as indicators of chloroquine resistance in isolates of $P$. falciparum obtained from Osogbo western Nigeria using a hybridization probe method on a Real Time PCR technology platform. The result of this study showed a high prevalence of $p f c r t \mathrm{~T} 76$ $(74 \%)$. This observation is consistent with the previous reports from various malaria endemic regions where chloroquine has been widely used. A significant association was also found between the overall in vivo rate of treat- 


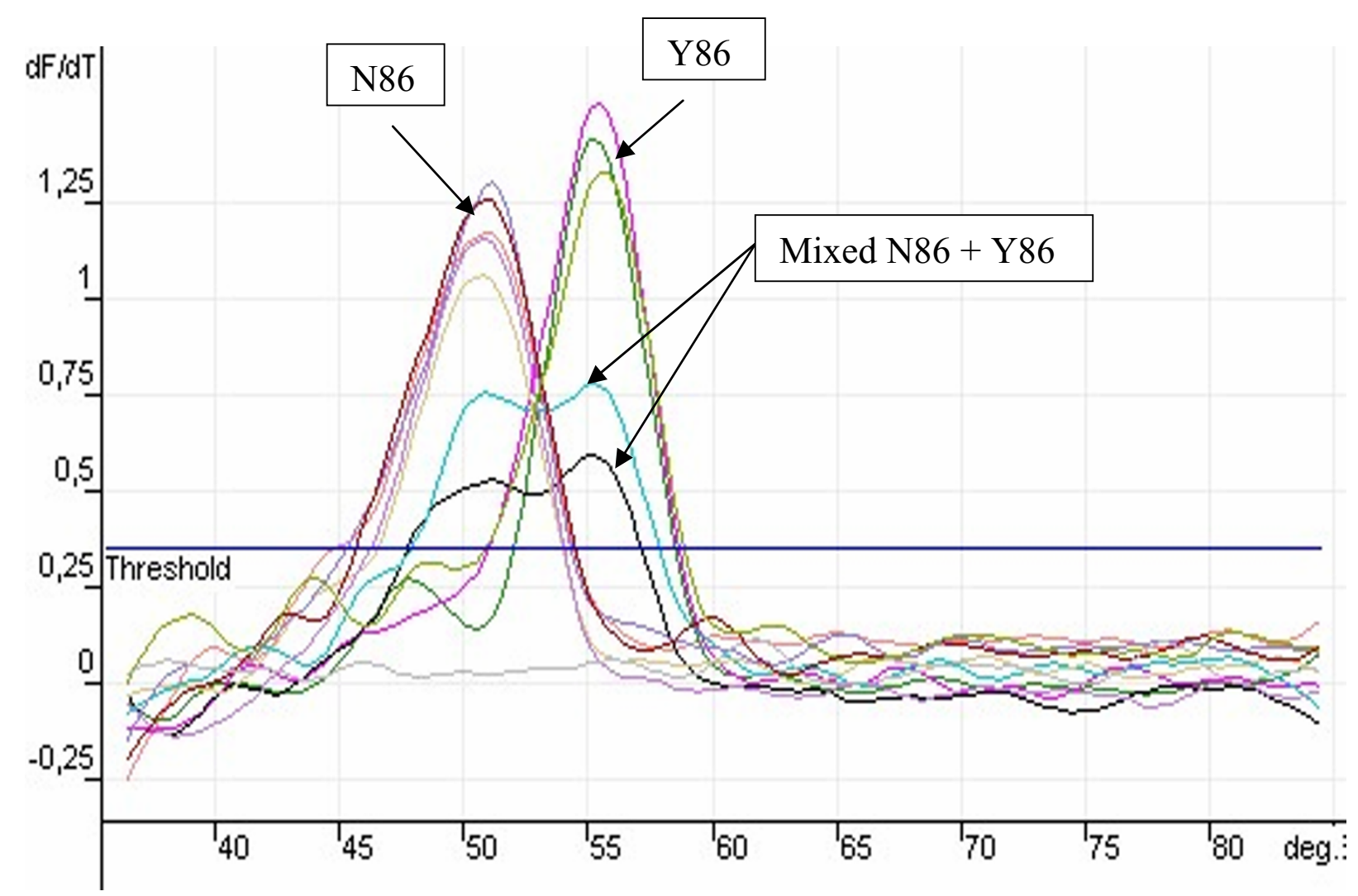

Figure 2

Example of a typical melting curve analysis generated by real time PCR of the Pfmdr position 86.

ment failure and the frequency of mutated $p f c r t$ gene in the population $(\mathrm{P}=0.004)$ as already shown previously in western Nigeria [19] and other malaria endemic areas [18]. 93\% of the pre-treatment isolates carried the pfcrt T76 and mixed allele while among the post treatment isolate the prevalence was $98 \%$. The facts that this polymorphism was present in all the recrudescence isolates emphasised again the fact that this mutation is important in CQR.

The point mutation of asparagine to tyrosine at codon 86 of $p f m d r 1$ has been associated with CQR in some studies $[8,9]$ but not in others [12]. In the present study, both the pfmdr1 Y86 and F184 mutations showed no correlation with resistance to chloroquine. Both the wild type and the mutant alleles for each locus were present in both sensitive and resistance isolates. Previously, an association was established between chloroquine-resistance and alleles of the $p f m d r 1$ gene in laboratory isolates obtained from different parts of the world [8]. Others had considered whether this association existed in parasite isolates obtained directly from the field $[9,20]$. Their analysis showed that African isolates predominantly possess polymorphism at two alleles, codon-86 and codon-184, with a positive association, although incomplete, between mutation of codon-86 and CQR. A similar association has been found in several studies $[4,11,21,22]$. Nevertheless, much confusion has surrounded the association of different pfmdr1 alleles to chloroquine resistance because numerous studies have had contradictory results. Transfection studies as well as carefully controlled molecular epidemiologic studies have shown that there are strong associations between $p f m d r 1$ polymorphisms and antimalarial resistance [7]. However, like many other studies [12] the present findings have failed to find such associations because the presence of both wild-type and mutant-type alleles in our samples were largely independent of their in vivo responses. Also the current belief that the combination of $p f c r t$ and $p f m d r 1$ polymorphisms result in higher levels of CQR [4] was not observed in this study. Although 
Table 3: Prevalence of pfcrt and pfmdrl alleles in post-treatment samples of Nigerian children with acute uncomplicated malaria.

\begin{tabular}{|c|c|c|c|c|}
\hline \multirow[t]{2}{*}{ Allele } & \multirow[t]{2}{*}{ Frequency } & \multicolumn{3}{|c|}{ Treatment outcome (\%) } \\
\hline & & Cured $\mathrm{N}=72$ & Failed $\mathrm{N}=44$ & P. value \\
\hline \multicolumn{5}{|l|}{$N=116$} \\
\hline Pfcrt T 76 & $86(74 \%)$ & $5 \mid(7 \mid \%)$ & 41 (93\%) & \\
\hline Pfort K 76 & II (I2\%) & II (I5\%) & $0(0 \%)$ & $0.003^{*}$ \\
\hline $\mathrm{T} 76+\mathrm{K} 76$ & $19(14 \%)$ & $10(14 \%)$ & $9(20 \%)$ & \\
\hline \multicolumn{5}{|l|}{$N=116$} \\
\hline Pfmdrl Y86 & $34(29 \%)$ & $22(31 \%)$ & $12(27 \%)$ & \\
\hline Pfmdrl N86 & $74(64 \%)$ & 47 (65\%) & $27(61 \%)$ & n.s. \\
\hline Y86+N86 & $8(7 \%)$ & $3(4 \%)$ & $5(11 \%)$ & \\
\hline \multicolumn{5}{|l|}{$N=116$} \\
\hline PfmdrI FI84 & $74(64 \%)$ & 45 (63\%) & $29(65 \%)$ & \\
\hline PfmdrI Y I84 & $35(30 \%)$ & $24(33 \%)$ & II (25\%) & n.s. \\
\hline FI84+YI84 & $7(6 \%)$ & $3(4 \%)$ & $4(9 \%)$ & \\
\hline \multicolumn{5}{|l|}{$N=116$} \\
\hline $\mathrm{T} 76+\mathrm{Y} 86$ & $37(32 \%)$ & $21(29 \%)$ & $16(36 \%)$ & \\
\hline $\mathrm{K} 76+\mathrm{N} 86$ & $7(6 \%)$ & $7(10 \%)$ & $0(0 \%)$ & $0.028^{*}$ \\
\hline Mixed & $72(62 \%)$ & $44(61 \%)$ & $28(64 \%)$ & \\
\hline \multicolumn{5}{|l|}{$N=116$} \\
\hline $\mathrm{T} 76+\mathrm{FI} 86$ & 73 (63\%) & $40(56 \%)$ & $33(75 \%)$ & \\
\hline$K 76+Y 184$ & $2(2 \%)$ & $2(3 \%)$ & $0(0 \%)$ & n.s. \\
\hline Mixed & $41(35 \%)$ & $30(42 \%)$ & II (25\%) & \\
\hline \multicolumn{5}{|l|}{$N=116$} \\
\hline $\mathrm{T} 76+\mathrm{Y} 86+\mathrm{F} 184$ & $35(30 \%)$ & $19(26 \%)$ & $16(36 \%)$ & \\
\hline $\mathrm{K} 76+\mathrm{N} 86+\mathrm{Y} 184$ & $3(3 \%)$ & $3(4 \%)$ & $0(0 \%)$ & n.s. \\
\hline Mixed & $78(67 \%)$ & $50(70 \%)$ & $28(64 \%)$ & \\
\hline
\end{tabular}

n.s. is not significant

a significant association was observed $(p=0.028)$, it was not in any way stronger than the one observed with $p f c r t$ alone. Analysis of altered gene expression and other mechanisms that may contribute to a resistant phenotype is needed before the role of $p f m d r 1$ can be excluded.
A recent report from Ibadan Nigeria a neighbouring town to Osogbo had suggested an association and linkage disequilibrium between the pfcrt T76 and pfmdr1 Y86 alleles in chloroquine-resistant isolates [19]. On the contrary this study suggested no possible association between these two polymorphic alleles and in vivo chloroquine resist-

Table 4: Prevalence of mutant pfmdrl/pfcrt genes in Day 0 and recrudescence samples of patients that failed chloroquine treatment

\begin{tabular}{|c|c|c|c|}
\hline \multicolumn{4}{|c|}{ Prevalence of alleles } \\
\hline Alleles & Day 0 & Recrudescence & Mc Nemar $P$ value \\
\hline T76 & $44 / 44(100 \%)$ & $43 / 44(98 \%)$ & 1 \\
\hline Y86 & I7/44 (39\%) & |8/44 (4|\%) & I \\
\hline FI84 & $33 / 44(75 \%)$ & $27 / 44(61 \%)$ & $0.031 *$ \\
\hline T76+Y86 & $44 / 44(100 \%)$ & $43 / 44(98 \%)$ & I \\
\hline T76+FI84 & $44 / 44(100 \%)$ & $43 / 44$ (98\%) & I \\
\hline $\mathrm{T} 76+\mathrm{Y} 86+\mathrm{F} 184$ & $44 / 44(100 \%)$ & $44 / 44(100 \%)$ & I \\
\hline
\end{tabular}

Mixed infection is considered as mutant

* Significant at $\mathrm{P}<0.05$ 
ance and that these molecular markers by themselves may not predict in vivo chloroquine resistance.

\section{Conclusion}

In summary, the results of this study give further evidence to the reliability of the 76T pfcrt point mutation as a molecular marker for CQ resistance. Conversely, the role of the Y86 and F184 point mutation of $p f m d r 1$ gene in CQ resistance remains elusive. The analysis of the relevant mutations by RT-PCR provides a rapid and reliable method for epidemiological and clinical studies suited for higher throughput.

\section{Authors' contributions}

OO performed the in vivo testing and the molecular analysis, and drafted the manuscript.

TOO performed the in vivo testing.

FR participated in the molecular study.

AFF-B supervised the design of the study.

PGK and JFJK supervised the molecular work and the interpretation of the data and helped to draft the manuscript.

All authors read and agreed to the content of the manuscript.

\section{Acknowledgements}

The authors are grateful to all parents and guardian who volunteered to participate in the study. We thank Titi Okewumi, Adeola Ayileka and Kumbi Akinwale for their assistance in the laboratory. We also thank Eric Kenjo for helping with the statistical analysis. We thank Ladoke Akintola University of Technology for their contribution to malaria research clinic and laboratory where the work was carried out. $O O$ is a recipient of a Deutscher Akademischer Austausch Dienst (DAAD) Fellowship.

\section{References}

I. Laufer MK, Thesing PC, Eddington ND, Masonga R, Dzinjalamala FK, Takala SL, Taylor TE, Plowe CV: Return of chloroquine antimalarial efficacy in Malawi. N Engl J Med 2006, 355:1959-I 966.

2. Fidock DA, Nomura T, Talley AK, Cooper RA, Dzekunov SM, Ferdig MT, Ursos LM, Sidhu AB, Naude B, Deitsch KW, Su XZ, Wootton JC, Roepe PD, Wellems TE: Mutations in the P. falciparum digestive vacuole transmembrane protein PfCRT and evidence for their role in chloroquine resistance. Mol Cell 2000, 6:86I-87I.

3. Severini C, Menegon M, Sannella AR, Paglia MG, Narciso P, Matteelli A, Gulletta M, Caramello P, Canta F, Xayavong MV, Moura IN, Pieniazek NJ, Taramelli D, Majori G: Prevalence of pfcrt point mutations and level of chloroquine resistance in Plasmodium falciparum isolates from Africa. Infect Genet Evol 2006, 6:262-268.

4. Babiker HA, Pringle SJ, Abdel-Muhsin A, Mackinnon M, Hunt P, Walliker D: High-level chloroquine resistance in Sudanese isolates of Plasmodium falciparum is associated with mutations in the chloroquine resistance transporter gene pfort and the multidrug resistance Gene pfmdrl. J Infect Dis 200I, | 83:1535-1538.
5. Vinayak S, Biswas S, Dev V, Kumar A, Ansari MA, Sharma YD: Prevalence of the K76T mutation in the pfcrt gene of Plasmodium falciparum among chloroquine responders in India. Acta Trop 2003, 87:287-293.

6. Djimde A, Doumbo OK, Cortese JF, Kayentao K, Doumbo S, Diourte Y, Dicko A, Su XZ, Nomura T, Fidock DA, Wellems TE, Plowe CV, Coulibaly D: A molecular marker for chloroquine-resistant falciparum malaria. N Engl J Med 200I, 344:257-263.

7. Duraisingh MT, Cowman AF: Contribution of the pfmdrl gene to antimalarial drug-resistance. Acta Trop 2005, 94: I8I-190.

8. Foote SJ, Kyle DE, Martin RK, Oduola AM, Forsyth K, Kemp DJ, Cowman AF: Several alleles of the multidrug-resistance gene are closely linked to chloroquine resistance in Plasmodium falciparum. Nature 1990, 345:255-258.

9. Basco LK, Le Bras J, Rhoades Z, Wilson CM: Analysis of pfmdr I and drug susceptibility in fresh isolates of Plasmodium falciparum from subsaharan Africa. Mol Biochem Parasitol 1995, 74:157-166.

10. Gomez-Saladin E, Fryauff DJ, Taylor WR, Laksana BS, Susanti AI, Purnomo, Subianto B, Richie TL: Plasmodium falciparum mdr I mutations and in vivo chloroquine resistance in Indonesia. Am J Trop Med Hyg 1999, 61:240-244.

II. Nagesha HS, Din S, Casey GJ, Susanti AI, Fryauff DJ, Reeder JC, Cowman AF: Mutations in the pfmdrI, dhfr and dhps genes of Plasmodium falciparum are associated with in-vivo drug resistance in West Papua, Indonesia. Trans $R$ Soc Trop Med Hyg 200I, 95:43-49.

12. Pillai DR, Labbe AC, Vanisaveth V, Hongvangthong B, Pomphida S, Inkathone S, Zhong K, Kain KC: Plasmodium falciparum malaria in Laos: chloroquine treatment outcome and predictive value of molecular markers. J Infect Dis 200I, I 83:789-795.

13. Thomas SM, Ndir O, Dieng T, Mboup S, Wypij D, Maguire JH, Wirth DF: In vitro chloroquine susceptibility and PCR analysis of pfcrt and pfmdrl polymorphisms in Plasmodium falciparum isolates from Senegal. Am J Trop Med Hyg 2002, 66:474-480.

14. WHO: Assessment of therapeutic efficacy of antimalarial drugs for uncomplicated malaria in areas with intense transmission. Geneva: World Health Organistion WHOIMAL/ 1996:96-I077.

15. Snounou G, Zhu X, Siripoon N, Jarra W, Thaithong S, Brown KN, Viriyakosol S: Biased distribution of $\mathbf{m s p l}$ and $\mathbf{m s p} 2$ allelic variants in Plasmodium falciparum populations in Thailand. Trans R Soc Trop Med Hyg 1999, 93:369-374.

16. Kearns AM, Guiver M, James V, King J: Development and evaluation of a real-time quantitative PCR for the detection of human cytomegalovirus. J Virol Methods 200I, 95:|2|-|3|.

17. de Monbrison F, Raynaud D, Latour-Fondanaiche C, Staal A, Favre S, Kaiser K, Peyron F, Picot S: Real-time PCR for chloroquine sensitivity assay and for pfmdr I-pfcrt single nucleotide polymorphisms in Plasmodium falciparum. J Microbiol Methods 2003, 54:39|-40I.

18. Binder RK, Borrmann S, Adegnika AA, Missinou MA, Kremsner PG, Kun JF: Polymorphisms in the parasite genes for pfort and pfmdr-I as molecular markers for chloroquine resistance in Plasmodium falciparum in Lambarene, Gabon. Parasitol Res 2002, 88:475-476.

19. Happi TC, Thomas SM, Gbotosho GO, Falade CO, Akinboye DO, Gerena L, Hudson T, Sowunmi A, Kyle DE, Milhous W, Wirth DF, Oduola AM: Point mutations in the pfort and pfmdr-I genes of Plasmodium falciparum and clinical response to chloroquine, among malaria patients from Nigeria. Ann Trop Med Parasitol 2003, 97:439-45I.

20. Adagu IS, Warhurst DC, Ogala WN, Abdu-Aguye I, Audu LI, Bamgbola FO, Ovwigho UB: Antimalarial drug response of Plasmodium falciparum from Zaria, Nigeria. Trans $\mathrm{R}$ Soc Trop Med Hyg 1995, 89:422-425.

21. Duraisingh MT, von Seidlein LV, Jepson A, Jones P, Sambou I, Pinder $M$, Warhurst DC: Linkage disequilibrium between two chromosomally distinct loci associated with increased resistance to chloroquine in Plasmodium falciparum. Parasitology 2000, I 2 I ( Pt I): I-7.

22. von Seidlein L, Duraisingh MT, Drakeley CJ, Bailey R, Greenwood BM, Pinder M: Polymorphism of the Pfmdrl gene and chloroquine resistance in Plasmodium falciparum in The Gambia. Trans $R$ Soc Trop Med Hyg 1997, 9 1:450-453. 